therapy. However, there are no widely accepted CS dosing regimens for LN. We aim to identify the CS treatment approaches employed by providers for newly diagnosed pediatric proliferative $\mathrm{LN}$ in response to common and challenging clinical scenarios.

Methods Pediatric rheumatologists and nephrologists attending the 2018 Childhood Arthritis and Rheumatology Research Alliance (CARRA) meeting participated in a working group addressing CS use in newly diagnosed pediatric LN. Participants responded to 3 scenarios in live polling and 12 questions of CS management in small groups. A post meeting survey was sent to each participant.

Results In total, 51 physicians participated in the working group and 25 answered the survey. Of the 51 participants, $42(82 \%)$ reported prednisone to be the oral CS of choice to treat newly diagnosed pediatric $\mathrm{LN}$ and $64.7 \%$ favored liquid prednisolone if swallowing pills is problematic. Once daily dosing was the preferred regimen $(15 / 25,60 \%)$ to help patients with adherence. Some $(8 / 25,32 \%)$ use a twice daily regimen for prednisone doses $>2 \mathrm{mg} / \mathrm{kg} /$ day or $60 \mathrm{mg}$. A 3-4 times daily regimen was considered for hospitalized patients with severe disease manifestations by 6/ $25,24 \%$. Factors leading to the use of intravenous (IV) pulse methylprednisolone during the initial 12 months of therapy for proliferative LN varied among physicians with life-threatening extra-renal organ involvement (24/25, $96 \%)$, worsening $(20 / 25,80 \%)$ or slow improvement $(14 /$ $25,56 \%)$ of $\mathrm{LN}$ and concerns for non-adherence with oral prednisone $(19 / 25,76 \%)$ being the main factors. Laboratory results prompting a CS dose change are shown in table 1 (Panels A/B). Side effects such as weight gain (20/ $25,80 \%)$, difficult to control blood pressure $(19 / 25,76 \%)$ or hyperglycemia $(21 / 25,84 \%)$ were reported as reasons to taper CS. In patients with inactive LN on mycophenolate mofetil, the extra-renal features that prompt an increase in CS are new/worsening neuropsychiatric disease $(24 / 25,96 \%)$, cardiac $(23 / 25,92 \%)$, or pulmonary involvement $(23 / 25,92 \%)$. In cases of non-adherence, all physicians would discuss reasons for non-adherence with $72 \%$ choosing to start/increase the frequency of IV steroids. Additional consensus formation results on the use of CS in pediatric proliferative LN are being developed and will be available at the time of the meeting.

Conclusions Prescribed CS dosing regimens vary widely in the U.S. when used for the treatment of children with proliferative LN. Decisions on initial CS dosing regimens and subsequent management strategies remain provider dependent.

Funding Source(s): None

\section{SYSTEMIC LUPUS ERYTHERMATOSUS IS A RISK FACTOR FOR ATRIAL FIBRILLATION: A NATION-WIDE, POPULATION-BASED STUDY}

Sang-yeob Yim*. Depaerment of Internal EDICINE

10.1136/lupus-2019-|sm.42
Background Cardiac involvement is in more than half of the patients with systemic lupus erythematosus (SLE). However, large scale studies on the prevalence of atrial fibrillation (AF) in this disease do not exist. We aimed to investigate the incidence and clinical significance of AF in SLE.

Methods Patients with SLE $(n=21,143$; mean age, 41.8 \pm 13.13 years; female, 90.38\%) without previous AF were selected from the Korean National Health Insurance Service National Sample Cohort database between 2008 and 2014. Age-and sex-matched controls $(n=105,715)$ were randomly sampled in a 5:1 ratio from the population of individuals without SLE from same database. Both cohorts were followed-up for incidental AF and death until 2015.

Results AF was newly detected in 481 (2.27\%) in SLE and $619(0.59 \%)$ controls (incidence: 3.692 and 0.941 per 1000 person-years, respectively). After multivariate adjustment, SLE were found to be at a higher risk of developing AF compared to controls (hazard ratio (HR), 2.84; 95\% confidence interval (CI), 2.50-3.23). On subgroup analysis, younger (age <40) patients showed higher incidence of AF. SLE with incidental AF had a higher mortality rate compared to patients without SLE with AF (HR, 2.35; 95\% CI 1.73-3.20) and SLE without AF (HR, 3.53; 95\% CI 2.844.39) after adjustment.

Conclusions SLE was an independent risk factor for AF development, especially in younger patients without previous AF, stressing the importance of cardiac assessment in this population. AF development in patients with SLE was associated with increased mortality.

Funding Source(s): Kaplan-Meyer curves of atrial fibrillation in SLE and non-SLE patients.
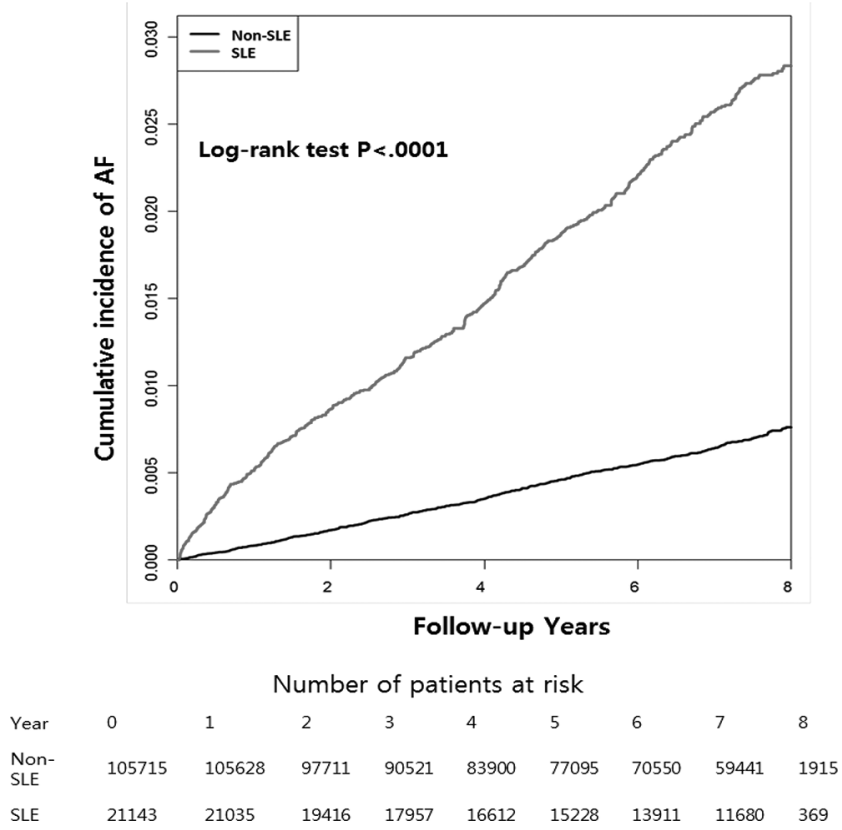

Abstract 42 Figure 1 Kaplan-Meyer curves showing incidence of atrial fibrillation in SLE and non-SLE patients. 